\title{
FEEDBACK PERFORMANCE AT THE STANFORD LINEAR COLLIDER ${ }^{\dagger}$
}

\author{
M.G. Minty, C. Adolphsen, L.J. Hendrickson, R. Sass, T. Slaton, M. Woodley \\ Stanford Linear Accelerator Center, Stanford University, Stanford, CA 94309 USA
}

Many feedback loops are used at the Stanford Linear Collider (SLC) to control the orbit and energy of particle beams. Problems with corrector magnet slew rates, actuator calibrations, and computation of the beam transport matrix between loops have resulted in operation of many SLC feedback loops at lower than design gain. The response of various feedback loops to these errors is measured and analyzed in an attempt to improve performance.

\section{INTRODUCTION}

The SLC feedback system [1,2] is a generalized, database driven system that applies state-space formalisms to stabilize the beam. Feedback has proven to be essential to the successful operation of the SLC. Problems encountered in running high current beams have forced us to adopt tighter tolerances on feedback control parameters and have motivated a stricter evaluation of feedback performance. Of particular concern is the fact that linac feedback loops are routinely operated with lower than design gain.

The design of each feedback loop relies on knowledge of the slew rates of the correctors used to cancel perturbations and of the noise spectrum of the beam. The effect of differences in corrector speeds has been measured and analyzed using high current beams. For a series of loops in succession correction for over compensation, or cascade [3], was found to be necessary, in which information from each loop is passed on to the loop immediately downstream. The transfer matrices between two consecutive cascaded loops are continuously updated, or adapted, using measurements of the natural beam jitter. Measurements of the performance of the adaptive cascade have shown sensitivity to the location of the noise source.

\section{SINGLE-LOOP RESPONSE}

The SLC beam rate is $120 \mathrm{~Hz}$, while most linac feedback loops sample the beam at $20 \mathrm{~Hz}$ due to CPU limitations The most downstream linac feedback loop, which is not cascaded; was upgraded to operate at $60 \mathrm{~Hz}$. However, when the sampling rate was increased, the measured beam jitter downstream of the loop increased.

${ }^{\dagger}$ Work supported by Department of Energy contract DE-AC03-76SF00515
The feedback gain, or the percentage of implemented change relative to the calculated change, was lowered as a result. A lower feedback gain implies less efficient correction.

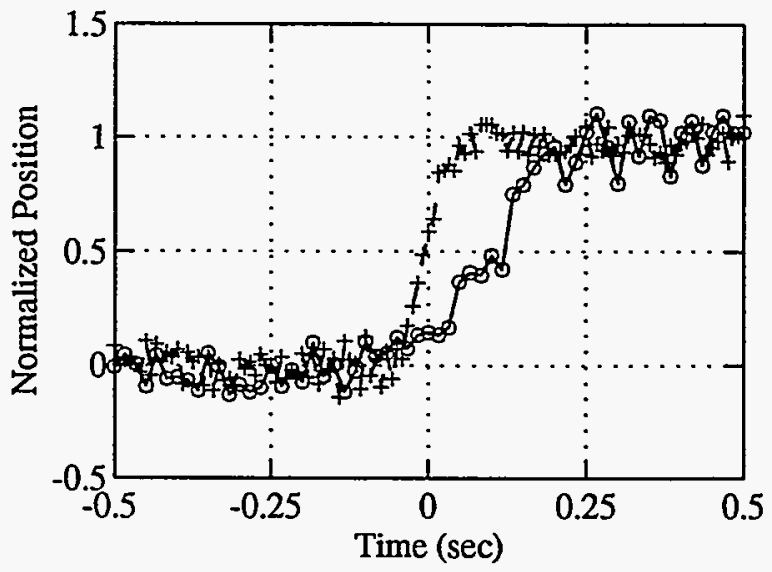

Figure 1: Slew rate measurement for a fast $(+)$ and a slow (o) corrector. A step function was applied to each corrector and the response of the beam as a function of time was measured on downstream BPMs.
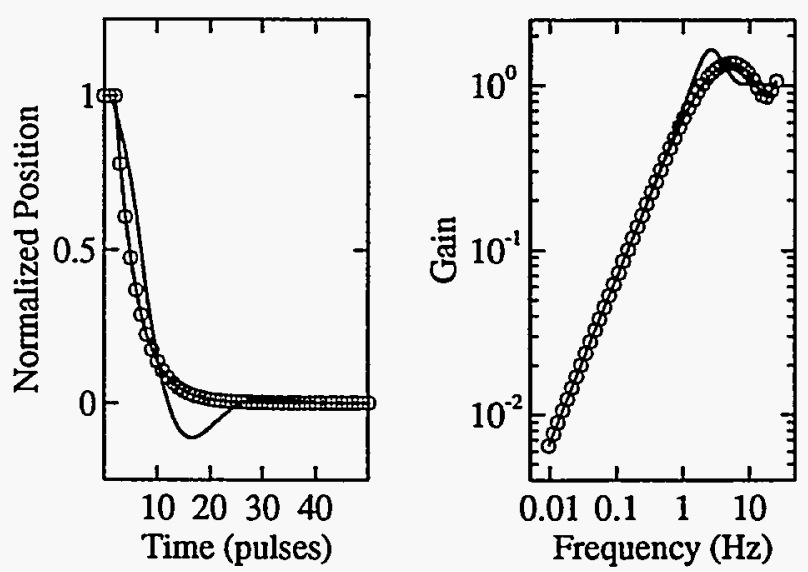

Figure 2: Time and frequency domain simulations of beam response to a step function assuming 8 fast (open circles) or 6 fast and 2 slow correctors (no circles).

Corrector slew rates were a suspected problem. If the feedback design assumed instantaneous correction, then a slow corrector could cause amplification of beam jitter. Slew rate measurements for feedback correctors revealed response times ranging from 7 to 12 beam pulses, compared to the design assumption of 6 pulses. Slew rate measurements are shown in Fig. 1 for two different feedback correctors. Plotted in Fig. 2 are time and frequency domain simulations for the $60 \mathrm{~Hz}$ feedback loop 
assuming ideal and mismatched correctors. These and similar studies show that slow or mismatched correctors cause feedback to overshoot. Notice also that the unity gain crossover point depends both on the feedback gain and on the corrector slew rates. Currently effort is being devoted to minimizing and matching corrector response times.

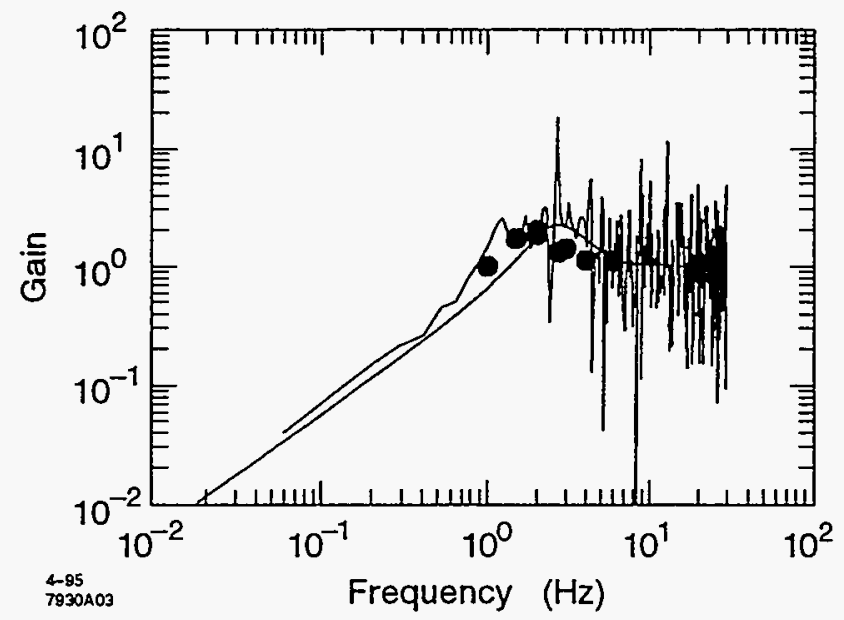

Figure 3: Simulated (smooth curve) and measured frequency response of a feedback loop. The filled circles were obtained from independent measurements using single frequency excitations of an upstream corrector.

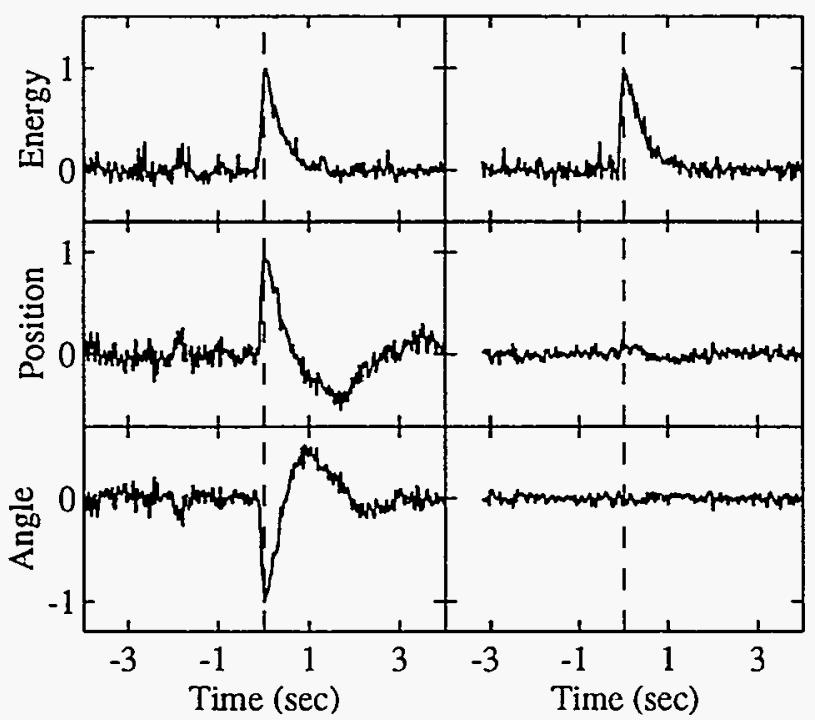

Figure 4: Normalized states computed by RTL feedback before (left) and after (right) including additional BPMs.

To quantify the single-loop response, a step function was applied to an upstream corrector and states were measured for the $60 \mathrm{~Hz}$ loop with feedback off, and with feedback on at unity gain. The data were then prepared for FFT analysis by inversion and reflection. The frequency response of the loop, which is given by the ratio of the FFTs of these data, is shown and compared with simulation in Fig. 3. The apparent noise at frequencies greater than about $5 \mathrm{~Hz}$ is a result of random noise in the measured states. Separate measurements using single frequency excitations of an upstream corrector are shown as filled circles. These data do not reproduce the occasional spikes observed in the FFT ratio. The solid line is a simulation which assumes that 2 of the 8 correctors are relatively slow. Comparison of Figs. 2 and 3 suggests that the observed structure in the undamped region of the response is due to slow or mismatched correctors.

In the linac, cooling water pumps generate beam jitter at frequencies near $59 \mathrm{~Hz}$. With $60 \mathrm{~Hz}$ feedback sampling, $59 \mathrm{~Hz}$ oscillations were aliased to near $1 \mathrm{~Hz}$. Depending on the loop gain, the feedback could amplify this $1 \mathrm{~Hz}$ oscillation. To avoid this, the feedback sampling rate was reduced to $40 \mathrm{~Hz}$. Ideally, such aliasing effects would best be avoided by sampling at the full beam rate.

Beam energy, as well as orbit angle and position, is measured and corrected in the two SLC damping-ring-tolinac (RTL) transport lines where there is dispersion. It was observed that these loops were having trouble distinguishing changes in beam energy from trajectory changes. This caused conflicting use of energy, angle, and position actuators by the feedback loops and oscillation of the beam. This problem was corrected by including additional beam position monitors (BPMs) in both dispersive and non-dispersive areas of the RTLs. Fig. 4 shows the feedback states computed in response to a step change in the beam ebergy before and after the addition of BPMs; note the improvement in the fitted angle and position (at a non-dispersive point).

\section{ADAPTIVE CASCADE}

In the current feedback control architecture, cascade passes information from each loop to its nearest downstream successor so that multiple correction of incoming perturbations is avoided. Each feedback loop in the series of loops should therefore correct only those perturbations generated immediately upstream. In adaptive cascade the transport matrix between successive feedback loops is continuously measured using natural beam jitter. Implicit to this procedure is the assumption that perturbations between two loops are uncorrelated with perturbations upstream of both loops. Cascaded feedback loops regulate states that have been adjusted by subtracting off corrections implemented by the nearest upstream loop. For a given loop within the cascade, the ratio of adjusted state to measured state defines the rejection ratio of the loop. This is the fraction of the upstream perturbation perceived by the loop to have been generated between it and its nearest upstream neighbor. Ideally the rejection 


\section{DISCLAIMER}

Portions of this document may be illegible in electronic image products. Images are produced from the best available original document. 
ratio should be zero, in which case the cascade is functioning properly. A nonzero rejection ratio indicates poor modeling of the transfer matrix between loops and results in over correction by the downstream loop.

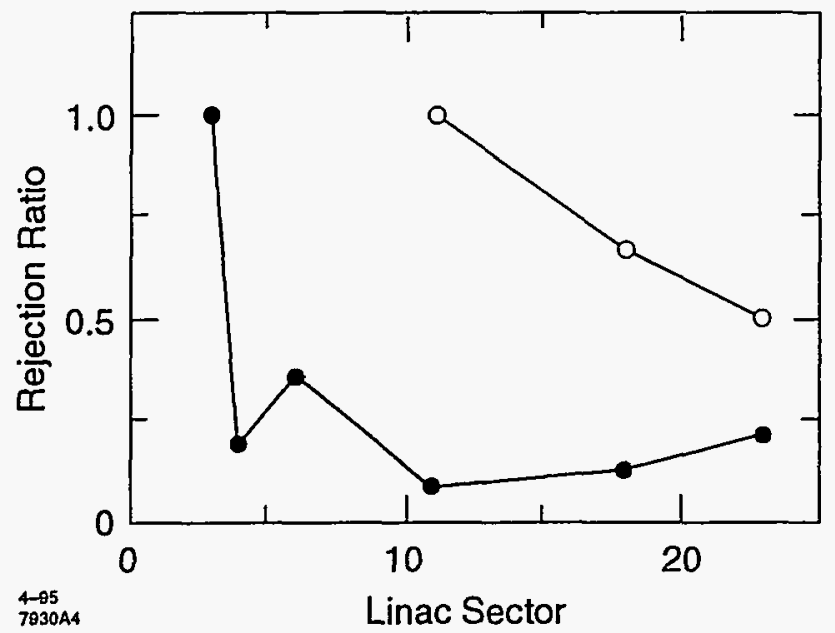

Figure 5: Rejection ratios measured with a pre-linac excitation (closed circles) and a mid-linac excitation (open circles).

Rejection ratio measurements are shown in Fig. 5. The data shown as closed circles were acquired by introducing a single step function perturbation upstream of the linac and measuring the response of all the cascaded feedback loops. These data and other measurements show that the rejection ratios varied from very good (10-20\%) to nearly non-cascaded, in which $50-100 \%$ of the perturbation was detected and corrected. Also shown as open circles are rejection ratios measured with an excitation made in the middle of the linac. The differences between these measurements indicate that the feedback response is sensitive to the location of the perturbation. A possible explanation for this is that the feedback design assumes linear transport matrices while at high current the transport matrices are actually nonlinear due to wake fields. Poor rejection is still not well understood.

\section{CALIBRATIONS AND MODELING}

Imperfections in the lattice model for the beam transport degrade feedback performance. Alternatively, the transport matrices can be measured. Using a recently improved calibration procedure, the position and angle states were measured as a function of feedback corrector magnet settings. Shown in Fig. 6 are measurements of the feedback response using the design SLC lattice model $(a, b)$ and using the calibration (c,d). While the regulation is undoubtedly improved, the time required for calibration (about 1 hour per loop) is significant. Comparison of the online calibration with the linac model is useful in isolating lattice errors. Long term stability of the calibration is coupled closely to the long term stability of the machine.
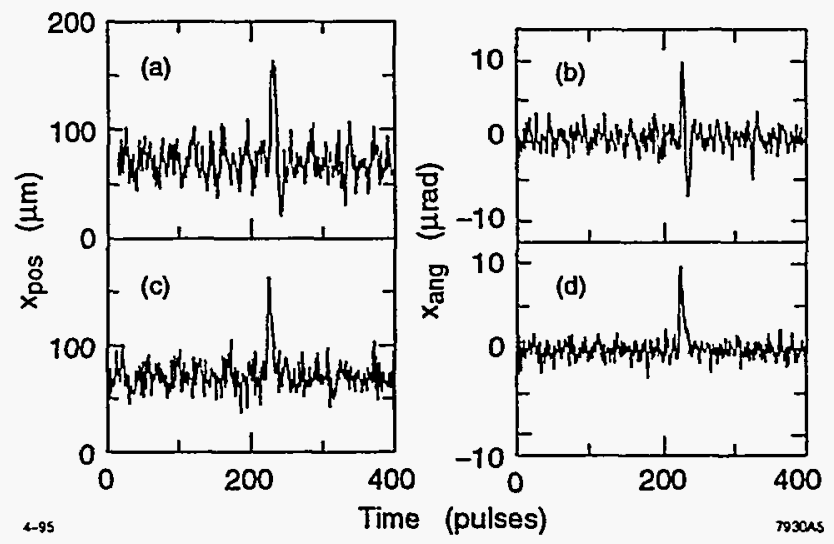

Figure 6: Feedback regulation with lattice model $(a, b)$ and with online calibration $(c, d)$. Feedback states are plotted as a function of time in response to an external excitation.

\section{CONCLUSION}

Operation of the linac feedback loops at lower than design gain has yet to be fully understood. Evaluations of corrector slew rates, sample rate aliasing, and cascade performance were presented. Future work will include analysis of the effect that wake fields have on beam transport, studying the significance of the location of noise sources, and evaluating how frequently loops should be calibrated.

\section{ACKNOWLEDGMENTS}

These studies were made possible by the dedicated work of the SLC controls group, who develop and maintain both the software and the hardware required for feedback. We also wish to thank Tom Himel for his helpful suggestions and support.

\section{REFERENCES}

[1] L.J. Hendrickson, et al., "Generalized Fast Feedback System in the SLC", ICALEPCS '91, Tsukuba, Japan, p. 414; SLAC-PUB-5683.

[2] F. Rouse, et al., "A Database Driven Fast Feedback System for the Stanford Linear Collider", Nucl. Inst. Meth. A316, 343 (1992); SLAC-PUB-5681.

[3] T. Himel, et al., "Adaptive Cascaded Beam Based Feedback at the SLC", IEEE PAC '93, Washington, DC, p. 2106; SLAC-PUB-6125. 


\section{DISCLAIMER}

This report was prepared as an account of work sponsored by an agency of the United States Government. Neither the United States Government nor any agency thereof, nor any of their employees, makes any warranty, express or implied, or assumes any legal liability or responsibility for the accuracy, completeness, or usefulness of any information, apparatus, product, or process disclosed, or represents that its use would not infringe privately owned rights. Reference herein to any specific commercial product, process, or service by trade name, trademark, manufacturer, or otherwise does not necessarily constitute or imply its endorsement, recommendation, or favoring by the United States Government or any agency thereof. The views and opinions of authors expressed herein do not necessarily state or reflect those of the United States Government or any agency thereof. 